Acta Crystallographica Section D

Biological

Crystallography

ISSN 0907-4449

Todd C. Appleby, Gary Larson, I. Wayne Cheney, Heli Walker, Jim Z. Wu, Weidong Zhong, Zhi Hong and Nanhua Yao*

Drug Discovery, Valeant Pharmaceuticals International, 3300 Hyland Avenue, Costa Mesa, CA 92626, USA

Correspondence e-mail: nyao@valeant.com

\title{
Structure of human uridine-cytidine kinase 2 determined by SIRAS using a rotating-anode $X$-ray generator and a single samarium derivative
}

Uridine-cytidine nucleoside kinase 2 (UCK2) is the ratelimiting enzyme in the pyrimidine-nucleotide salvage pathway. UCK2 catalyzes the phosphorylation of the natural ribonucleosides cytidine and uridine to cytidine $5^{\prime}$-monophosphate (CMP) and uridine $5^{\prime}$-monophosphate (UMP), respectively, and activates several important frontline antimetabolite drugs. The present contribution reports the rapid crystal structure determination of human UCK2 complexed with a magnesium ion and the reaction products adenosine $5^{\prime}$-diphosphate (ADP) and CMP. Diffraction data were collected on a copper rotating-anode X-ray generator from one native UCK2 crystal and a single samarium-derivative crystal. Utilizing the relatively high anomalous signal from the samarium derivative at the $\mathrm{Cu} K \alpha$ wavelength, the structure was determined by single isomorphous replacement and single anomalous signal (SIRAS) phasing techniques. Two of the four major samarium sites are located in the active sites of the two UCK2 molecules that form the asymmetric unit and appear to displace the magnesium ions present in the native crystals. The crystal structures of UCK 2 alone and in complex with various ligands have recently been determined using traditional multiple isomorphous replacement (MIR) phasing techniques and data from three heavy-atom derivatives. The reported structures validate our independently determined structure. Of more than 1000 kinase crystal structure entries in the Protein Data Bank, less than $1 \%$ of them have been determined by SIRAS. For the published kinase crystal structures determined by SIRAS, all data were reportedly collected at various synchrotron-radiation facilities. This study demonstrates that diffraction data collected from a single samarium derivative using $\mathrm{Cu} K \alpha$ radiation provides sufficient phasing power to determine a novel macromolecular crystal structure.

\section{Introduction}

The ability to phase macromolecular structures based solely on the presence of anomalous scatterers has been well established (Wang, 1985; Hendrickson, 1991) and can significantly reduce the time required to achieve a solution compared with traditional multiple isomorphous replacement (MIR) techniques. The need for anomalous data collected at multiple wavelengths has also been lessened, as improved instrumentation and methods for fast accurate data collection together with robust phasing algorithms have allowed the use of single-wavelength anomalous dispersion (SAD) as a viable alternative for structure determination (Dauter \& Dauter, 1999; Rice et al., 2000; Langs et al., 2001; Wang et al., 2004).
Received 14 October 2004 Accepted 10 December 2004

PDB Reference: human uridine-cytidine kinase 2 , 1 xrj, r1xrjsf. 
Furthermore, success in extracting the weak anomalous signal from diffraction data collected away from the absorption edge of a particular scatterer when utilizing fixed-wavelength conventional X-ray sources has reduced the need to travel to tunable-wavelength synchrotron beamlines in order to solve novel crystal structures by anomalous phasing techniques (Chen et al., 1991; Dauter, 2002; Evans \& Bricogne, 2002; Debreczeni et al., 2003; Yang et al., 2003). The requirement for the presence of an anomalous scatterer still remains, however, and obtaining a suitable derivative continues to be a bottleneck for many structure-determination projects. In addition, the type of derivative that is ultimately obtained generally dictates the data-collection and phasing strategies employed to solve the structure of the macromolecule.

Although its use is not widespread in the crystallographic literature (Flaherty et al., 1993; Grobler et al., 1996; Tsuge et al., 1999; Jeong et al., 2001; Head et al., 2003; Love et al., 2004), the lanthanide metal samarium $(\mathrm{Sm})$ has properties that make it desirable for use as an anomalously scattering ion in crystal structure determination. Firstly, as an ion and not part of a larger heavy-atom complex, Sm can diffuse rapidly into macromolecular crystals without having to form specific and stable bonds with a protein. This property allows short highconcentration soaks of native crystals similar to the successful procedures described for halogen derivitization (Dauter et al., 2000). In addition, samarium is expected to behave similarly to more physiologically relevant metal cations such as magnesium and calcium to the extent that the Sm ions would be likely to be chelated by acidic protein residues on the solventexposed surfaces, at the interfaces and in the active sites of crystallized proteins. Furthermore, although $\mathrm{Sm}$ does not have an absorption edge at the $\mathrm{Cu} K \alpha$ wavelength $(1.5418 \AA)$, it does have an $L_{\mathrm{I}}$ absorption edge $(1.6025 \AA)$ in the vicinity. In fact, the anomalous scattering contribution $\left(f^{\prime \prime}\right)$ of samarium at $1.5418 \AA$ is still considerable, with $f^{\prime \prime}$ approximately equal to 12.10 e. For comparison, the $f^{\prime \prime}$ of selenium at its $K$ absorption peak $(0.9793 \AA)$ is 3.85 e, while the $f^{\prime \prime}$ of sulfur at $1.5418 \AA$ is 0.56 e. Both selenium and sulfur have been used at these respective wavelengths to successfully phase numerous protein structures. We propose Sm soaking as a rational approach for introducing anomalous scatterers into macromolecular crystals for utilization in in-house SAD and SIRAS phasing experiments using $\mathrm{Cu} K \alpha$ radiation, especially in cases where the macromolecule of interest is known to bind metal cations or requires the presence of metals in the crystallization buffer.

Uridine-cytidine nucleoside kinase (UCK; EC 2.7.1.48) is the rate-limiting enzyme in the pyrimidine-nucleotide salvage pathway (Anderson et al., 1973). UCK catalyzes the phosphorylation of the natural ribonucleosides cytidine and uridine to CMP and UMP, respectively. CMP and UMP are subsequently phosphorylated by mono- and diphosphate kinases to form the $5^{\prime}$-triphosphate nucleoside substrates utilized in DNA and RNA synthesis (Parks et al., 1973; Van Rompay et al., 1999). Purified recombinant uridine-cytidine kinase 2 has been shown to efficiently utilize both ATP and GTP as phosphate donors for the reaction (Van Rompay et al.,
2001). Recently, the cloning of human UCK1 (277 residues) and UCK2 (261 residues) were reported. Although they share about $70 \%$ sequence identity, the precise physiological role of UCK1 remains unknown, as UCK2 is responsible for the majority of uridine and cytidine phosphorylation in cells (Koizumi et al., 2001; Van Rompay et al., 2001). UCK2 is also overexpressed in some human cancer cells. In fact, the tumorselective cytotoxicity of $3^{\prime}$ - $C$-ethynylcytidine (ECyd), a novel ribonucleoside analog that is especially effective against solid tumors such as those found in liver and pancreatic cancers (Hattori et al., 1996), probably results from activation by high levels of UCK2 in those tumor cells. This hypothesis is supported by evidence that cancer cells resistant to ECyd have defects in UCK2 activity (Tanaka et al., 1997). It has also been reported that UCK2 catalyzes the important initial phosphorylation step of cytotoxic nucleoside analog prodrugs such as cyclopentenylcytidine (Kang et al., 1989) and 5-fluorouridine (Greenberg et al., 1977). Efficient phosphorylation of these nucleoside prodrugs by UCK2 may play an important role in establishing the efficacy of these therapeutic agents. Thus, UCK2 is a target enzyme for antitumor and antiviral prodrug design. The fact that UCK2 overexpression is observed in rapidly dividing cells, including several tumor cell lines, suggests that it may also be an important anticancer target.

While this manuscript was in preparation, Suzuki et al. (2004) published the crystal structures of human UCK2 alone and in complex with the substrate cytidine, the feedback inhibitors CTP and UTP and the reaction products CMP and ADP. The authors describe the structural basis for specificity, catalysis and regulation of the enzyme in detail. The structure of the enzyme-product complex (PDB code 1uj2) is nearly identical to the independently determined UCK2 structure presented here.

Of about 1040 entries of kinase crystal structures in the Protein Data Bank, only ten have been determined by SIRAS techniques. In addition, data from synchrotron-radiation sources were used to determine nine of these ten particular structures. The only kinase entry (PDB code $1 \mathrm{~s} 4 \mathrm{q}$ ) that reports utilizing SIRAS techniques with data collected from a conventional rotating-anode $\mathrm{X}$-ray source currently awaits publication and the experimental details of the structure determination are therefore not yet available. The present report will focus on the strategies and techniques employed that resulted in the rapid structure determination of UCK2.

\section{Materials and methods}

\subsection{Expression, purification and crystallization}

Expression, purification and crystallization of the modified human UCK2 utilized in this study have been previously reported (Suzuki et al., 2003). Briefly, UCK2 with a deleted C-terminus (residues 251-260) was expressed with glutathione-S-transferase fused at the $\mathrm{N}$-terminus. A sequence of affinity, gel-filtration and ion-exchange chromatography steps yielded highly purified UCK2. The protein was concentrated 
to approximately $12 \mathrm{mg} \mathrm{ml}^{-1}$ and was stored at $193 \mathrm{~K}$. Diffraction-quality crystals were grown using the sitting-drop vapor-diffusion technique. Drops $(3 \mu \mathrm{l})$ were formed by mixing $1 \mu \mathrm{l}$ protein solution with $1 \mu \mathrm{l}$ ligand solution $(20 \mathrm{mM}$ cytidine, $10 \mathrm{~m} M \mathrm{MgCl}_{2}, 5 \mathrm{~m} M$ ATP) and $1 \mu \mathrm{l}$ reservoir solution (17-18\% PEG 8000, 0.2-0.3 M calcium acetate, $100 \mathrm{mM}$ Tris- $\mathrm{HCl} \mathrm{pH}$ 7.0). The drops were equilibrated against $0.5 \mathrm{ml}$ reservoir solution. Although UCK2 was crystallized in the presence of substrates (ATP and cytidine), the structure actually revealed an enzyme-product complex (UCK2-ADP$\mathrm{CMP}$ ), indicating that the reaction had occurred during the crystallization process. Block-shaped UCK2-ADP-CMP crystals generally appear in $3 \mathrm{~d}$ and grow for approximately one week, reaching dimensions of $0.2 \times 0.2 \times 0.15 \mathrm{~mm}$. The UCK2-ADP-CMP crystals belong to the monoclinic space group $C 2$, with unit-cell parameters $a=88.6, b=109.3$, $c=64.5 \AA, \beta=95.0^{\circ}$. The Matthews coefficient ( $V_{\mathrm{M}}$; Matthews, $1968)$ is $2.75 \AA^{3} \mathrm{Da}^{-1}$ assuming two monomers of approximately $28300 \mathrm{Da}$ each in the asymmetric unit, which corresponds to a solvent content of approximately $54 \%$.

\subsection{Derivative preparation}

Native UCK2 crystals were harvested from the mother liquor and transferred to the heavy-atom solution (19-20\% PEG 8000, 0.15 $M$ samarium acetate, $100 \mathrm{~m} M$ Tris- $\mathrm{HCl} \mathrm{pH}$ 7.0). After $1 \mathrm{~h}$, these crystals were transferred briefly to a cryosolution consisting of the heavy-atom solution supplemented with $20 \%$ glycerol and then frozen directly in a stream of nitrogen at $100 \mathrm{~K}$. The Sm-derivative crystals also belong to the monoclinic space group $C 2$, with unit-cell parameters $a=88.5, b=108.9, c=64.4 \AA, \beta=94.7^{\circ}$, and are isomorphous with respect to the native UCK2-ADP-CMP crystals.

\subsection{Data collection}

All diffraction data were collected using an in-house RUH3R copper rotating anode producing $\mathrm{Cu} K \alpha$ radiation and equipped with an R-AXIS IV ${ }^{++}$image-plate area detector mounted on a $2 \theta$ stage (Rigaku MSC, The Woodlands, TX, USA). The X-ray beam was focused by an Osmic Blue confocal optic and a collimator with a $0.6 \mathrm{~mm}$ rear aperture and a $0.3 \mathrm{~mm}$ front aperture (Rigaku MSC). The generator power settings were $50 \mathrm{kV}$ and $100 \mathrm{~mA}$ for all diffraction experiments. The experimental setup contained a single vertical spindle axis ( $\varphi$ axis). Owing to the reasonable unit-cell parameters of the UCK2-ADP-CMP crystals and the largearea format of the image-plate detector, all diffraction data were measured with the direct beam centered on the detector. Analyzing the initial images collected on the native crystal using the STRATEGY module of the software program CrystalClear (Rigaku MSC), it was determined that the unique crystallographic axis ( $b$ axis) was $30^{\circ}$ from the $\varphi$ axis and that $\sim 98 \%$ of a complete $2.0 \AA$ resolution data set could be collected using a continuous $\varphi$-axis scan of $150^{\circ}\left(1^{\circ}\right.$ per image) with a crystal-to-detector distance of $150 \mathrm{~mm}$ without reorienting the crystal. The exposure time selected to give strong signal out to the full range of resolution was $60 \mathrm{~min}$ per image.
Table 1

Structure determination and model refinement statistics.

(a) Crystal characteristics and data-collection statistics. Values for the outer resolution shell are given in parentheses.

\begin{tabular}{|c|c|c|}
\hline & Native & Sm derivative \\
\hline Unit-cell parameters $\left(\AA,^{\circ}\right)$ & $\begin{array}{c}a=88.6, b=109.3 \\
c=64.5, \beta=95.0\end{array}$ & $\begin{array}{r}a=88.5, b=108.9 \\
\quad c=64.4, \beta=94.7\end{array}$ \\
\hline Space group & $C 2$ & $C 2$ \\
\hline Molecules per AU & 2 & 2 \\
\hline X-ray source & Rigaku RUH3R & Rigaku RUH3R \\
\hline Wavelength (§) & 1.5418 & 1.5418 \\
\hline Resolution $(\AA)$ & $100.0-2.0(2.07-2.00)$ & $100.0-2.5(2.59-2.50)$ \\
\hline No. of observations & 120872 & 156169 \\
\hline No. of unique reflections & 40772 & $40316 \dagger$ \\
\hline Completeness (\%) & $98.8(96.2)$ & $97.9(96.4)$ \\
\hline Mean $I / \sigma(I)$ & $22.0(6.5)$ & $21.1(4.7)$ \\
\hline$R_{\text {merge }}$ & $0.046(0.165)$ & $0.062(0.295)$ \\
\hline
\end{tabular}

(b) Crystallographic data and refinement statistics. The FOM from the $C C P 4$ programs MLPHARE and DM is the average figure-of-merit $\simeq\langle\cos$ (phase error) $)$.

\begin{tabular}{ll}
\hline MLPHARE FOM & $\begin{array}{c}0.344(50.0-2.5 \AA \text { resolution }) \\
\text { for } 21067 \text { reflections }\end{array}$ \\
DM FOM & $0.747(50.0-2.5 \AA$ resolution $)$ \\
& for 21067 reflections \\
Resolution range $(\AA)$ & $50.0-2.0$ \\
No. of reflections & $45293(40732$ working set, \\
& 4561 test set $)$ \\
Completeness (\%) & 94.1 \\
No. of protein residues/atoms & $424 / 3395$ \\
No. of ligand atoms & 98 \\
No. of water atoms & 315 \\
$R_{\text {cryst }}$ & 0.209 \\
$R_{\text {free }}$ & 0.237 \\
Root-mean-square deviations & \\
$\quad$ Bond lengths $(\AA)$ & 0.006 \\
Bond angles $\left({ }^{\circ}\right)$ & 1.00 \\
Ramachandran plot statistics: residues in & \\
$\quad$ Most favored regions & $367(94.8 \%)$ \\
Additional allowed regions & $18(4.7 \%)$ \\
Generously allowed regions & $2(0.5 \%)$ \\
Disallowed regions & $0(0.0 \%)$ \\
\hline
\end{tabular}

$\dagger$ Bijvoet pairs are treated as unique reflections for the Sm-derivative data. $\ddagger R_{\text {merge }}=$ $\sum_{h k l} \sum_{i}|I-\langle I\rangle| / \sum_{h k l} \sum_{i} I$.

CrystalClear analysis of initial images collected on the $\mathrm{Sm}$ derivative crystal revealed that the $b$ axis was nearly perpendicular to ( $86^{\circ}$ from) the $\varphi$ axis, while the $a$ and $c$ axes were more than $10^{\circ}$ off of the spindle axis. Thus, $97 \%$ of a complete $2.5 \AA$ resolution data set could be collected using a continuous $\varphi$-axis scan. In order to collect Bijvoet pairs and to maximize redundancy, the SAD data were collected on the Sm derivative using $1^{\circ}$ oscillations per image for a total of $360^{\circ}$ with a crystal-to-detector distance of $180 \mathrm{~mm}$ and exposure times of 25 min per image. All data were integrated and scaled using DENZO and SCALEPACK (Otwinowski \& Minor, 1997) (Table 1). For the Sm-derivative data, anomalous pairs were treated as non-equivalent reflections and were kept separate during scaling and merging steps by using the 'SCALE ANOMALOUS' flag in the program SCALEPACK. The refined mosaicity and average data redundancy were $0.46^{\circ}$ and 3.0, respectively, for the native crystal and $0.61^{\circ}$ and 3.9 (for Bijvoet measurements), respectively, for the Sm-derivative crystal. 


\subsection{Heavy atom and structure determination}

The anomalous signal present in the diffraction data is represented graphically in Fig. 1, where the Bijvoet ratio $\left\langle\Delta F^{\text {anom }}\right\rangle /\langle F\rangle$ is plotted as a function of resolution. The anomalous differences from the Sm-derivative data were used to determine the heavy-atom positions in the crystal. Normalized structure-factor magnitude differences (diff $E \mathrm{~s}$ ) were generated from the Bijvoet pairs using the DREAR package (Blessing \& Smith, 1999). The anomalously scattering substructure was determined by the dual-space directmethods (Shake-and-Bake) procedure (Weeks et al., 1994) using the computer program $\operatorname{SnB}$ (v.2.2; Weeks \& Miller, 1999). 4000 triple invariants were generated from the largest $400 \operatorname{diff} E$ values. A total of 1000 trials were processed, each starting with ten random atoms and each run for 20 cycles of phase refinement. A trace of the minimal function $\left(R_{\min }\right)$

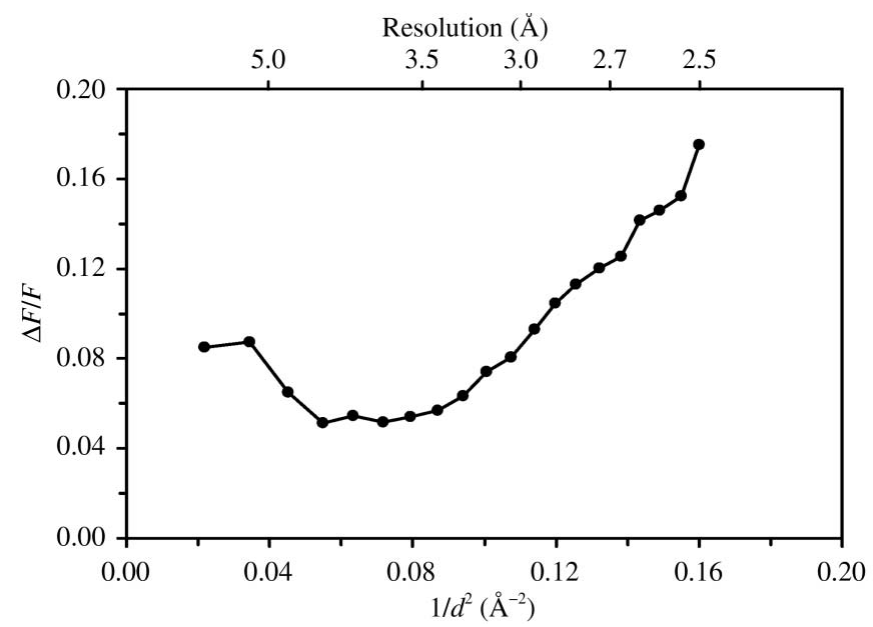

Figure 1

The presence of anomalous signal in the diffraction data. The Bijvoet ratio $\left\langle\Delta F^{\text {anom }}\right\rangle /\langle F\rangle$ is plotted as a function of resolution for the data collected on the UCK2-ADP-CMP samarium-derivative crystal.
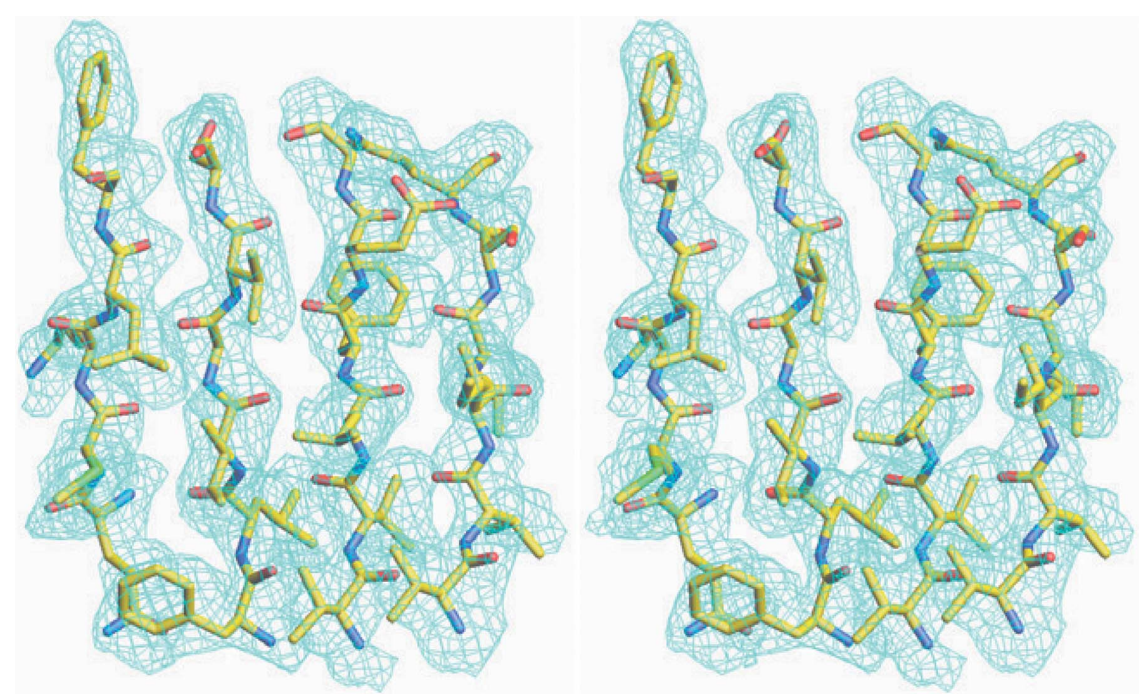

Figure 2

A stereo diagram of the averaged solvent-flattened electron-density map calculated from SIRAS phases to $2.5 \AA$ resolution. The map is superimposed on coordinates of the final model in the region of the core $\beta$-sheet. revealed a bimodal distribution. The density values of the top four peaks from the best solution were 20.2, 16.9, 15.4 and 13.9 , respectively. The next six density peaks in the list range in height from 7.8 to 6.1 . These results suggested that the top four density peaks represented four major Sm-atom positions in the asymmetric unit. These four sites were visualized graphically. An approximate twofold non-crystallographic symmetry (NCS) axis was identified by generating several symmetryrelated sites for the four Sm atoms and then grouping specific atoms into NCS pairs based on visual inspection using the interactive molecular-graphics program $O$ (Jones et al., 1991). NCS pairs were then superimposed using the $L S Q \_E X P L I C I T$ routine in $O$. This program generates a transformation matrix that can later be used directly in density-modification programs for the purpose of NCS averaging. Heavy-atom parameter refinement and phasing were performed with the MLPHARE program (Otwinowski, 1991) from the $C C P 4$ software suite (Collaborative Computational Project, Number 4, 1994) and the phases were improved by solvent flattening using the program DM (Cowtan \& Zhang, 1999). The correct hand for the Sm-atom model was determined by inspecting the resulting electron-density maps. The initial average figure-of-merit (FOM) for all reflections to $2.5 \AA$ resolution is 0.344 and improves to 0.735 after density modification (Table 1). Utilizing the available twofold NCS averaging in conjunction with solvent flattening resulted in a final FOM of 0.747 . This averaged experimental map was of excellent quality, showing clear main-chain and side-chain density (Fig. 2) as well as several solvent molecules. On the basis of this map, a $\mathrm{C}^{\alpha}$ trace for the first molecule (molecule $A$ ) was generated manually, followed by the addition of mainchain and side-chain atoms using the molecular-graphics program $O$ (Jones et al., 1991). Molecule $B$ was then generated by the NCS transformation obtained from the Sm sites. After rigid-body refinement and one round of torsion-angle molecular-dynamics refinement (Rice \& Brünger, 1994) using the program CNX (Accelrys, San Diego, CA, USA), the $R$ factor dropped to $28 \%$ with a free $R$ factor of $32 \%$ using data to $2.5 \AA$ resolution. The model was further improved by iterative cycles of torsionangle annealing using the $2.0 \AA$ resolution native data set and manual adjustment to the model. In later rounds of refinement, ADP, CMP, a magnesium ion and several water molecules were added to both molecules in the asymmetric unit on the basis of difference density and reasonable hydrogen-bonding distances and geometries.

\section{Results and discussion}

\subsection{Structure of human UCK2}

Our UCK2 structure is nearly identical to that of the product-bound structure 
determined previously by Suzuki and coworkers (PDB code 1uj2; Suzuki et al., 2004). Both structures crystallize in space group $C 2$ with similar unit-cell parameters that vary by less than $0.6 \%$. Superposition of the two structures reveals an r.m.s.d. of $0.15 \AA^{2}$ for 1696 protein backbone atoms in the asymmetric unit (ASU). In contrast, the r.m.s.d. for 844 backbone atoms of molecule $A$ is $0.54 \AA^{2}$ when superimposed with molecule $B$ within the ASU of our structure. A tetramer

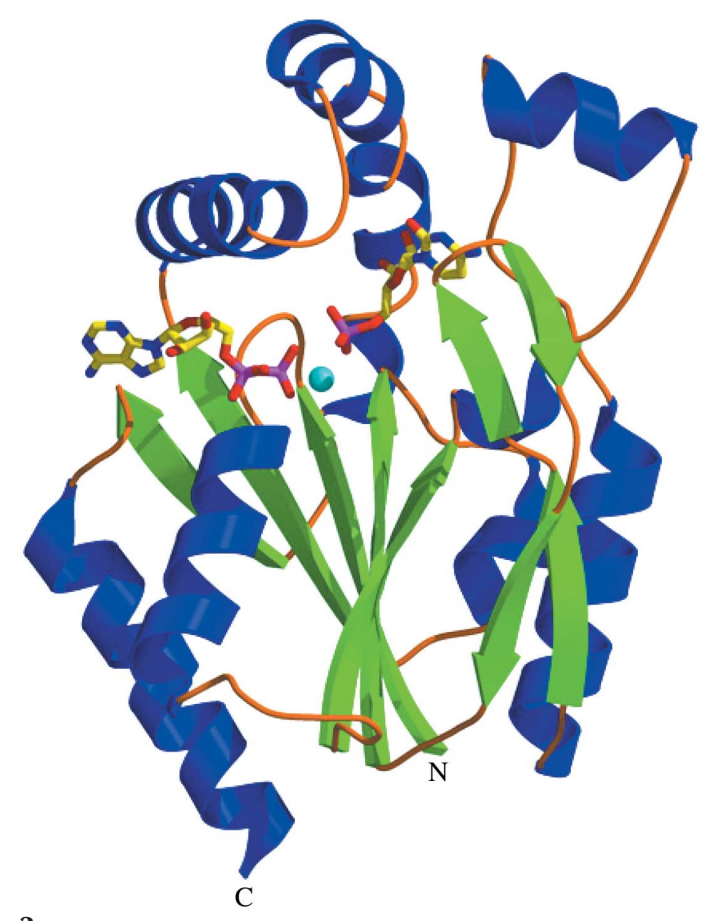

Figure 3

The overall structure of the human UCK2 monomer. Strands (green) and helices (blue) are represented by arrows and coils, respectively. The reaction products ADP and CMP are shown as sticks, while the magnesium ion is denoted by a cyan sphere. $\mathrm{N}$ - and C-termini are labeled accordingly.

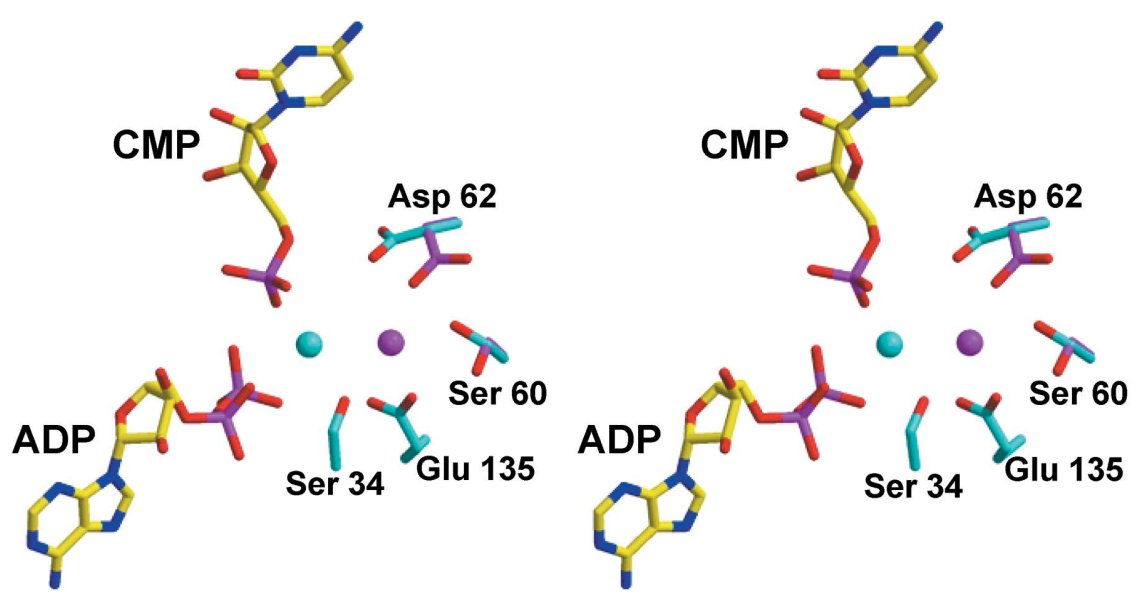

Figure 4

A stereoview comparing the active sites of the two molecules in the asymmetric unit. Asp62 and Ser60 with alternate side-chain conformations together with the magnesium ion from molecule $B$ (magenta) are superimposed on the protein side chains and the magnesium ion from molecule $A$ (cyan). Stick models for the reaction products ATP and CMP from molecule $A$ are also displayed. is formed by the two molecules in the ASU and two additional molecules generated by crystallographic symmetry. The UCK2 monomer contains a core five-stranded parallel $\beta$-sheet flanked on both sides by a pair of $\alpha$-helices (Fig. 3). Three additional $\alpha$-helices sit above the core-sheet structure and together with a large $\beta$-hairpin loop complete the active site. The enzyme active site contains the products of the phosphate-transfer reaction, ADP and CMP. Since the reaction substrates were added to the crystallization drops, we assume that the reaction proceeded during crystallization. The $\beta$-hairpin loop of UCK2 forms an extensive portion of the deep nucleoside-binding pocket. The positions of this loop in our ligand-bound structure and in the structure of the apo enzyme (PDB code 1ufq; Suzuki et al., 2004) suggest that this unique structural element might act as a flap that modulates substrate binding and product release. A total of 12 hydrogen bonds are formed between side chains of UCK2 and the CMP molecule, accounting for much of the enzyme's specificity. His117 and Tyr112 allow UCK2 to select for nucleosides with both cytosine and uracil bases by either accepting hydrogen bonds from or donating hydrogen bonds to the 4-amino group of cytidine or the 6-oxo group of uridine, respectively. The 2'and $3^{\prime}$-hydroxyl groups of the ribose moiety are involved in hydrogen bonding to both the acidic side chain of Asp84 and the guanidinium group of Arg166. These four hydrogen bonds most likely explain why UCK2 selects for ribonucleoside over deoxyribonucleoside substrates (Van Rompay et al., 2001). As previously reported (Suzuki et al., 2004), the carboxylate of Asp62 is positioned near the $5^{\prime}-\mathrm{O}$ atom of the nucleoside monophosphate product and most likely acts as a general base to activate the $5^{\prime}$-hydroxyl group of the nucleoside substrate, thus facilitating attack of the $\gamma$-phosphate of ATP. A similar role for an acidic residue acting as a catalytic base has also been proposed for other sugar and nucleoside kinases, including Asp255 in ribokinase (Sigrell et al., 1998), Asp300 in adenosine kinase (Mathews et al., 1998) and, more recently, Glu53 in deoxycytidine kinase (Sabini et al., 2003).

In addition to the presence of nucleotides, the active sites of both UCK2 molecules in the asymmetric unit also contain one magnesium ion. In molecule $A$, the magnesium ion is coordinated by the 5 '-phosphate of CMP and the $\beta$-phosphate of ADP in addition to Ser34 and two water molecules. The relatively weaker electron density in this region of the active site of molecule $B$ suggests a lower occupancy for magnesium and ADP. Interestingly, the magnesium ion in molecule $B$ appears to be slightly removed from the active site $(\sim 3 \AA)$ and is coordinated by the side chains of Glu135 and Ser34 as well as the side chain of the catalytic base Asp62, which displays an alternate side-chain conformation compared with that in molecule $A$ (Fig. 4). It is possible that after phosphate 

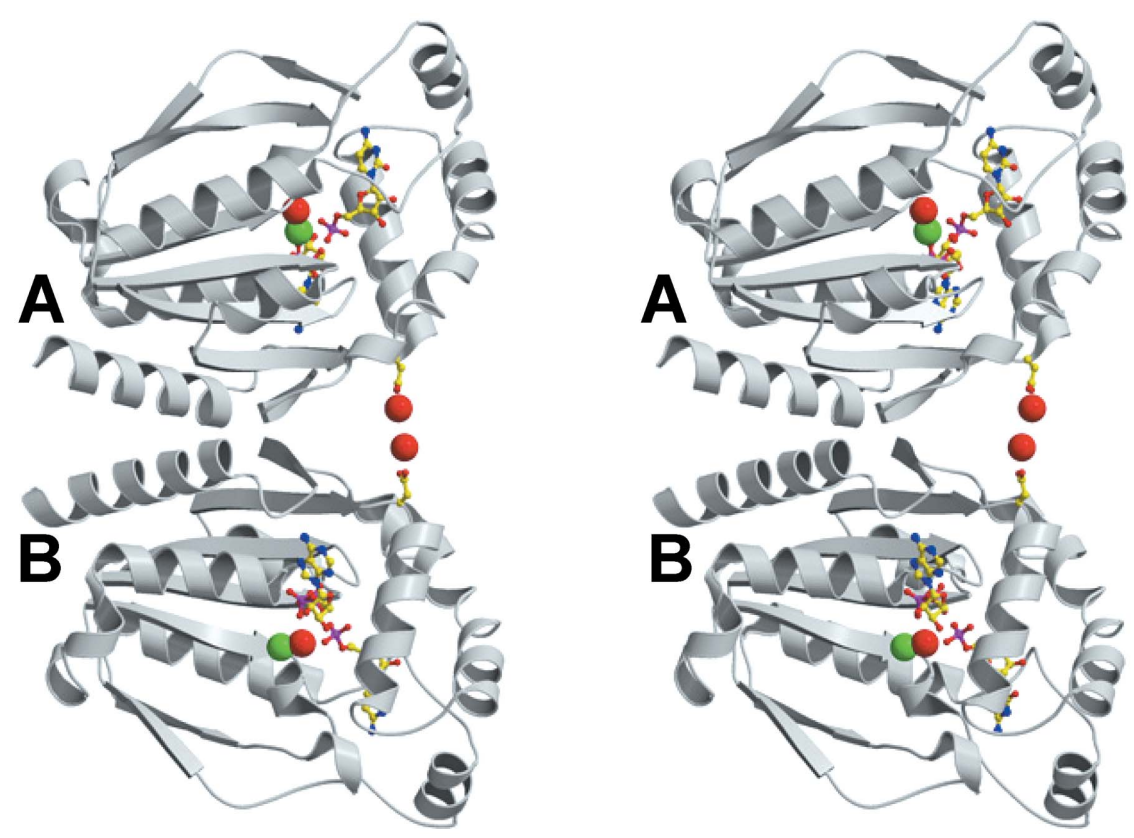

Figure 5

A stereoview indicating the location of the four major samarium sites (red spheres). Positions for the magnesium ions in the native UCK2-ADP-CMP crystals are shown as green spheres. ADP, CMP and Asp158 are shown as ball-and-stick models. Molecules $A$ and $B$ are labeled accordingly.

transfer the solvent-exposed ADP molecule diffuses out of the active site, while the acidic side chain of Asp62 flips away from the 5'-phosphate of the newly formed CMP molecule. The magnesium ion may remain associated with the Asp62 carboxylate during this process. If this is the case, molecule $B$ may represent an intermediate conformation of the enzyme that occurs during the product-release stage of the reaction. In fact, we have observed that the longer the UCK2-ADP-CMP crystals are allowed to sit in the mother liquor before harvesting the weaker the density for ADP becomes, while the difference density indicating the conformation change of Asp62 becomes stronger (data not shown).

\subsection{Heavy-atom positions}

The brief high-concentration samarium acetate soak resulted in the addition of four well ordered Sm atoms per asymmetric unit of the UCK2-ADP-CMP crystals (Fig. 5). Two of the four samarium sites lie at the interfaces between crystallographically related molecules (one between $A$ and $A_{\text {symm }}$, one between $B$ and $B_{\text {symm }}$ ) near the center of the UCK2 tetramer. The positively charged samarium ion is positioned between Asp158 of one molecule and Glu194 of its crystallographic symmetry mate. The remaining two Sm-atom positions are located within the active sites of the UCK2 molecules, where they appear to have displaced the magnesium ions which are required for crystallization. The Sm atoms are coordinated by the $5^{\prime}$-monophosphate of CMP and the side chain of Asp62. Of the remaining six peaks found by $S n B$, four also correlate with peaks in the anomalous difference Fourier map calculated using amplitudes of $\Delta F^{\text {anom }}$ and phases calculated from the four major $\mathrm{Sm}$ sites. These four peaks are probably minor Sm sites and are near surface exposed acidic residues of UCK2 in the crystals. Including the four additional sites does allow a slight improvement in the phasing statistics, with the averaged solvent-flattened FOM improving from 0.747 to 0.765 . The quality of the new map, however, reveals no major improvements over the initial map calculated using the four major sites.

\subsection{Conclusions}

Soaking native UCK2 crystals in $0.15 M$ samarium acetate for $1 \mathrm{~h}$ resulted in a heavy-atom derivative with four major $\mathrm{Sm}$ sites in the asymmetric unit. As with any heavy-atom soaking experiment, the type of salt, the concentration of the compound and the duration of soaking will require some optimization for each unique structuredetermination project. The fact that magnesium was required for crystallization and was subsequently present in the product complex may have improved the chances that ordered $\mathrm{Sm}$ sites would result from the heavy-atom soaking procedure. The presence of additional well ordered sites on the surface of the UCK2 molecules does indicate that samarium ions have the potential to generate useful heavy-atom derivatives for a diverse set of protein crystals. Further studies are required to substantiate the general application of this technique.

Owing to their large $f^{\prime \prime}$ values, the lanthanide metals are a useful class of heavy atoms for anomalous diffraction studies. Gadolinium (Gd), another lanthanide metal, has already shown great utility in macromolecular structure determination by SAD techniques using conventional laboratory X-ray sources (Girard et al., 2003). The anomalous signal at the $\mathrm{Cu} K \alpha$ wavelength arising from the presence of the Sm atoms in our study was sufficient to determine the heavy-atom substructure by direct methods and provided sufficient phasing power to generate an interpretable electron-density map. These results suggest that the use of samarium may represent a general strategy for obtaining a useful heavy-atom derivative for in-house structure determination using SAD and SIRAS phasing techniques. This strategy should definitely be considered when working with metal-binding proteins or proteins that require the presence of metal ions for crystallization.

The authors acknowledge Todd Vo and Jason Amsbaugh for their assistance in cloning and purifying human UCK2.

\section{References}

Anderson, R. G., Douglas, L. J., Hussey, H. \& Baddiley, J. (1973). Biochem. J. 136, 871-876. 
Blessing, R. H. \& Smith, G. D. (1999). J. Appl. Cryst. 32, 664670.

Chen, L. Q., Rose, J. P., Breslow, E., Yang, D., Chang, W. R., Furey, W. F. Jr, Sax, M. \& Wang, B.-C. (1991). Proc. Natl Acad. Sci. USA, 88, $4240-4244$.

Collaborative Computational Project, Number 4 (1994). Acta Cryst. D50, 760-763.

Cowtan, K. D. \& Zhang, K. Y. (1999). Prog. Biophys. Mol. Biol. 72, 245-270.

Dauter, Z. (2002). Curr. Opin. Struct. Biol. 12, 674-678.

Dauter, Z. \& Dauter, M. (1999). J. Mol. Biol. 289, 93-101.

Dauter, Z., Dauter, M. \& Rajashankar, K. R. (2000). Acta Cryst. D56, 232-237.

Debreczeni, J. É., Bunkóczi, G., Ma, Q., Blaser, H. \& Sheldrick, G. M. (2003). Acta Cryst. D59, 688-696.

Evans, G. \& Bricogne, G. (2002). Acta Cryst. D58, 976-991.

Flaherty, K. M., Zozulya, S., Stryer, L. \& McKay, D. B. (1993). Cell, 75 , 709-716.

Girard, E., Stelter, M., Vicat, J. \& Kahn, R. (2003). Acta Cryst. D59, 1914-1922.

Greenberg, N., Schumm, D. E. \& Webb, T. E. (1977). Biochem. J. 164, 379-387.

Grobler, J. A., Essen, L. O., Williams, R. L. \& Hurley, J. H. (1996). Nature Struct. Biol. 3, 788-795.

Hattori, H., Tanaka, M., Fukushima, M., Sasaki, T. \& Matsuda, A. (1996). J. Med. Chem. 39, 5005-5011.

Head, J. F., Mealy, T. R., McCormack, F. X. \& Seaton, B. A. (2003). J. Biol. Chem. 278, 43254-43260.

Hendrickson, W. A. (1991). Science, 254, 51-58.

Jeong, S.-T., Kim, H.-K., Kim, S.-J., Pan, J.-G., Oh, T.-K. \& Ryu, S.-E. (2001). Acta Cryst. D57, 1300-1302.

Jones, T. A., Zou, J.-Y., Cowan, S. W. \& Kjeldgaard, M. (1991). Acta Cryst A47, 110-119.

Kang, G. J., Cooney, D. A., Moyer, J. D., Kelley, J. A., Kim, H. Y., Marquez, V. E. \& Johns, D. G. (1989). J. Biol. Chem. 264, 713718.

Koizumi, K., Shimamoto, Y., Azuma, A., Wataya, Y., Matsuda, A., Sasaki, T. \& Fukushima, M. (2001). Int. J. Mol. Med. 8, 273278.

Langs, D. A., Blessing, R. H. \& Guo, D. (2001). Acta Cryst. A57, 240 243.
Love, R. A., Maegley, K. A., Yu, X., Ferre, R. A., Lingardo, L. K., Diehl, W., Parge, H. E., Dragovich, P. S. \& Fuhrman, S. A. (2004). Structure, 12, 1533-1544.

Mathews, I. I., Erion, M. D. \& Ealick, S. E. (1998). Biochemistry, 37, 15607-15620.

Matthews, B. W. (1968). J. Mol. Biol. 33, 491-497.

Otwinowski, Z. (1991). Proccedings of the CCP4 Study Weekend. Isomorphous Replacement and Anomalous Scattering, edited by W. Wolf, P. R. Evans \& A. G. W. Leslie, pp. 80-86. Warrington: Daresbury Laboratory.

Otwinowski, Z. \& Minor, W. (1997). Methods Enzymol. 276, 307-326.

Parks, R. E. Jr, Brown, P. R., Cheng, Y. C., Agarwal, K. C., Kong, C. M., Agarwal, R. P. \& Parks, C. C. (1973). Comp. Biochem. Physiol. B, 45, 355-364.

Rice, L. M. \& Brünger, A. T. (1994). Proteins, 19, 277-290.

Rice, L. M., Earnest, T. N. \& Brunger, A. T. (2000). Acta Cryst. D56, 1413-1420.

Sabini, E., Ort, S., Monnerjahn, C., Konrad, M. \& Lavie, A. (2003). Nature Struct. Biol. 10, 513-519.

Sigrell, J. A., Cameron, A. D., Jones, T. A. \& Mowbray, S. L. (1998). Structure, 6, 183-193.

Suzuki, N. N., Koizumi, K., Fukushima, M., Matsuda, A. \& Inagaki, F. (2003). Acta Cryst. D59, 1477-1478.

Suzuki, N. N., Koizumi, K., Fukushima, M., Matsuda, A. \& Inagaki, F. (2004). Structure, 12, 751-764.

Tanaka, M., Tabata, S., Matsuda, A., Fukushima, M., Eshima, K. \& Sasaki, T. (1997). Gan To Kagaku Ryoho, 24, 476-482.

Tsuge, H., Nagahama, M., Nishimura, T., Sakaguchi, Y., Katunuma, N. \& Sakurai, J. (1999). J. Struct. Biol. 126, 175-177.

Van Rompay, A. R., Johansson, M. \& Karlsson, A. (1999). Mol. Pharmacol. 56, 562-569.

Van Rompay, A. R., Norda, A., Linden, K., Johansson, M. \& Karlsson, A. (2001). Mol. Pharmacol. 59, 1181-1186.

Wang, B.-C. (1985). Methods Enzymol. 115, 90-112.

Wang, J. W., Chen, J. R., Gu, Y. X., Zheng, C. D., Jiang, F., Fan, H. F., Terwilliger, T. C. \& Hao, Q. (2004). Acta Cryst. D60, 1244-1253.

Weeks, C. M., DeTitta, G. T., Hauptman, H. A., Thuman, P. \& Miller, R. (1994). Acta Cryst. A50, 210-220.

Weeks, C. M. \& Miller, R. (1999). J. Appl. Cryst. 32, 120-124.

Yang, C., Pflugrath, J. W., Courville, D. A., Stence, C. N. \& Ferrara, J. D. (2003). Acta Cryst. D59, 1943-1957. 